OPEN ACCESS

Edited by:

Yasuo Yanagi,

Yokohama City University Medical

Center, Japan

Reviewed by:

Carlo Gesualdo,

Università della Campania Luigi Vanvitelli, Italy

Beatrice Gallo,

Moorfields Eye Hospital, NHS Foundation Trust, United Kingdom

*Correspondence:

Zhanyu Zhou

zhouzhanyu1125@126.com

tThese authors have contributed equally to this work

Specialty section:

This article was submitted to Ophthalmology

a section of the journal

Frontiers in Medicine

Received: 15 April 2021 Accepted: 25 June 2021 Published: 20 July 2021

Citation:

Tong N, Wang L, Wang N and Zhou Z

(2021) Bilateral Diffuse Uveal

Melanocytic Proliferation Secondary to

Rectal Adenocarcinoma: A Case

Report and Literature Review.

Front. Med. 8:691686

doi: 10.3389/fmed.2021.691686

\section{Bilateral Diffuse Uveal Melanocytic Proliferation Secondary to Rectal Adenocarcinoma: A Case Report and Literature Review}

\author{
Nianting Tong ${ }^{\dagger}$, Liangyu Wang ${ }^{\dagger}$, Nan Wang and Zhanyu Zhou * \\ Department of Ophthalmology, Qingdao Municipal Hospital, Qingdao, China
}

Background: Bilateral diffuse uveal melanocytic proliferation (BDUMP) is a rare paraneoplastic intraocular syndrome that causes progressive visual loss in patients, and is associated with an underlying malignancy. Recently, the incidence of BDUMP has increased with the prolonged life expectancy of oncology patients.

Case Presentation: We report a case of a 68-year-old man with significant visual loss in both eyes. The patient presented with a diffusely thickened choroid and ciliary body, extremely shallow anterior chamber, increased intraocular pressure, and cataract formation, accompanied by exudative retinal detachment in both eyes. He underwent a pars plana vitrectomy and choroidal biopsy, which revealed benign proliferation of melanocytes. A small amount of subretinal fluid persisted, and uveal thickness persisted in the early postoperative period. During the 1-year follow-up assessment, he underwent rectal tumor resection, and was pathologically diagnosed with rectal adenocarcinoma. Six months after the rectal tumor resection, the subretinal fluid was completely absorbed and the retina had reattached. The thickness of both the ciliary body and choroid had significantly decreased.

Conclusion: This case report describes a rare paraneoplastic intraocular syndrome, BDUMP, which was associated with rectal adenocarcinoma. Treatment for the primary malignancy gradually improved the visual symptoms and signs.

Keywords: bilateral diffuse uveal melanocytic proliferation, case report, paraneoplastic syndrome, exudative retinal detach, rectal adenocarcinoma

\section{INTRODUCTION}

Bilateral diffuse uveal melanocytic proliferation (BDUMP), first described by Machemer (1), is a rare paraneoplastic syndrome affecting the eyes. Although the incidence of BDUMP was previously extremely low, with about 1.15 cases per year from 1980 to 2000, the incidence increased to 4.4 cases per year around 2017 because of increased disease awareness and an increased life expectancy among oncology patients (2). Ocular findings in BDUMP patients include multiple red-orange patches at the level of the retinal pigment epithelium (RPE) with early hyperfluorescence according to fluorescein angiography (FA), diffuse thickening of the uveal tract, rapidly progressive cataract, and exudative retinal detachment (3-6). BDUMP is caused by a diffuse proliferation of benign 


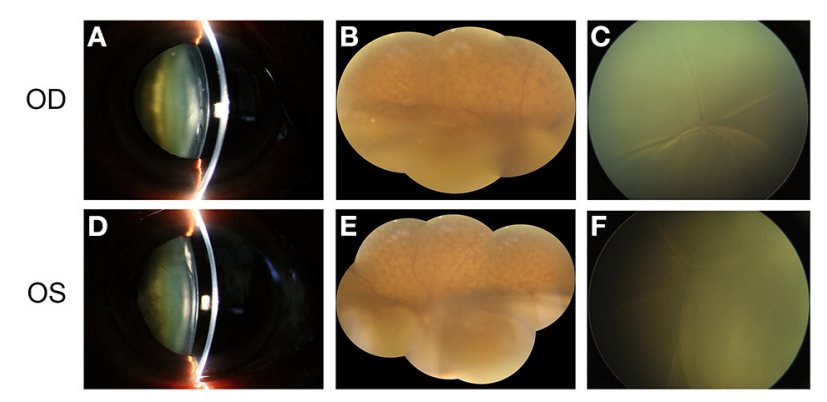

FIGURE 1 | The main clinical manifestation of the patient before surgery. (A) shows the obvious cataract in the right eye of the patient. (B) shows the exudative retinal detachment in the inferior part of the retina, accompanied by naevus-like multifocal reddish patches in the superior part of the fundus. Total retinal detachment was observed in the posterior pole of the retina when the patient was in the supine position (C). (D-F) show the similar clinical manifestation of the left eye.

melanocytes in the uvea, predominantly in the choroid, and is histopathologically unrelated to the primary non-ocular tumor (3).

BDUMP usually occurs in patients with systemic malignant disease, specifically, ovarian carcinoma in women $(4,7-10)$ and lung carcinoma in men $(3,4,11-13)$. Occasionally, BDUMP is associated with gastric adenocarcinoma $(14,15)$, colonic adenocarcinoma (16), bladder cancer (5, 17, 18), pancreatic carcinoma (19), or primary vitreoretinal lymphoma $(20,21)$.

We herein report a case of BDUMP. To the best of our knowledge, this is the first report of BDUMP associated with rectal adenocarcinoma. We present the results of comprehensive ophthalmic analyses and a detailed pathological examination.

\section{CASE PRESENTATION}

A 68-year-old Chinese man complained of gradual visual loss that occurred in both eyes over a period longer than 9 months. Approximately 6 months before he came to our clinic, he underwent a subtotal pneumonectomy to treat a pulmonary tumor, for which the pathological diagnosis was benign. Two months before he came to our clinic, assessments at another medical center revealed increased intraocular pressure (IOP) (around $30 \mathrm{mmHg}$ in both eyes), a whole circle thickened ciliary body according to ultrasonic biomicroscopy (UBM), and decreased anterior chamber depth. He was able to count fingers in an assessment of best corrected visual acuity (BCVA) for both eyes. The IOP was $19 \mathrm{mmHg}$ for the right eye and $20 \mathrm{mmHg}$ for the left eye. A slit-lamp examination of the anterior segment of the eyes showed obviously tortuous and dilated episcleral vessels (Supplementary Figure 1), significantly decreased depth of anterior chamber, iris bombe, slight eversion of iris pigment at the pupillary margin and prominent cortical opacity of the lens (Figure 1). There were no signs of synechia, neovascular membranes in the iris, or active inflammation (Figure 1). Open angles with pigmentation within the trabecular meshwork were revealed by gonioscopy. Color

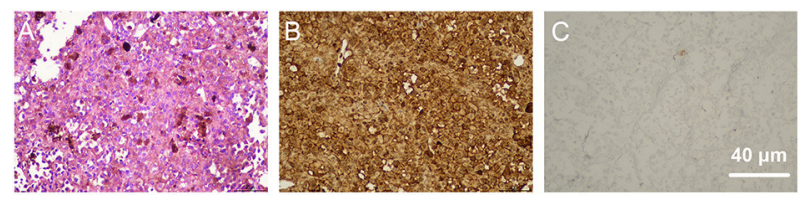

FIGURE 2 | Pathological examination of biopsies collected from the choroid during surgery. (A) shows hematoxylin-eosin (HE) staining of the biopsies, which revealed a predominant spindle-shaped melanocytic cell mass. Immunohistochemical staining showed that the tissue was positive for antibodies against S-100 (B) and negative for antibodies against Ki67 (C).

fundus retinal photography revealed typical exudative retinal detachment in both eyes (Figure 1), accompanied by naevuslike multifocal reddish patches under RPE in the superior part of the fundus. Ocular coherence tomography (OCT) showed exudative retinal detachment of the macula with multiple hyperreflective elevated lesions under RPE. Lensectomy, peripheral iridectomy, diagnostic pars plana vitrectomy (PPV), and silicon oil tamponade were performed on the right eye to deepen the anterior chamber, restore the transparency of the refractive media, reattach the retina, and take a choroid sample for biopsy. Vitreous fluid and subretinal fluid were collected during the surgery, but immunological analyses revealed no specific positive results. Haematoxylin-eosin (HE) staining for biopsies from the choroid suggested predominantly spindleshaped melanocytic cell masses, while immunohistochemistry showed that the samples were positive for antibodies against S100, and negative for antibodies against Ki67, LCA, PAX-5, and SOX-10 (Figure 2). The histopathological examination showed that the choroid lesion was a benign proliferation of melanocytes.

One month after surgery in the right eye, the patient underwent surgery in the left eye. This had a similar procedure but no biopsy, and subretinal fluid was drained through the sclera. At a 1-week follow-up assessment, the BCVA of the patient had recovered to $20 / 100$ for the right eye and 20/80 for the left eye, and the IOP was $21 \mathrm{mmHg}$ for both eyes. The depth of the anterior chamber had increased and the iris bombe had disappeared (Figure 3). The retina was basically reattached with only a small amount of subretinal fluid. A mass of multifocal reddish patches (Figure 3) was found all around the retina, especially in the mid-peripheral sections. These patches were consistent with the elevated hyper-reflective mass under RPE (Figure 3) in the OCT data, and also with the hyperfluorescence in the autofluorescence images and hypofluorescence in the early FA data.

A small amount of subretinal fluid persisted during the early postoperative follow-up period, and this was accompanied by thickening of the choroid. Three months after the operation, the patient underwent a rectectomy to treat a rectal tumor, and postoperative pathology revealed a rectal adenocarcinoma. Six months after the rectectomy, the subretinal fluid had completely disappeared and the thickness of the ciliary body and choroid had significantly decreased (Figure 4). The BCVA had improved to $20 / 60$ for both eyes at the last follow-up. 

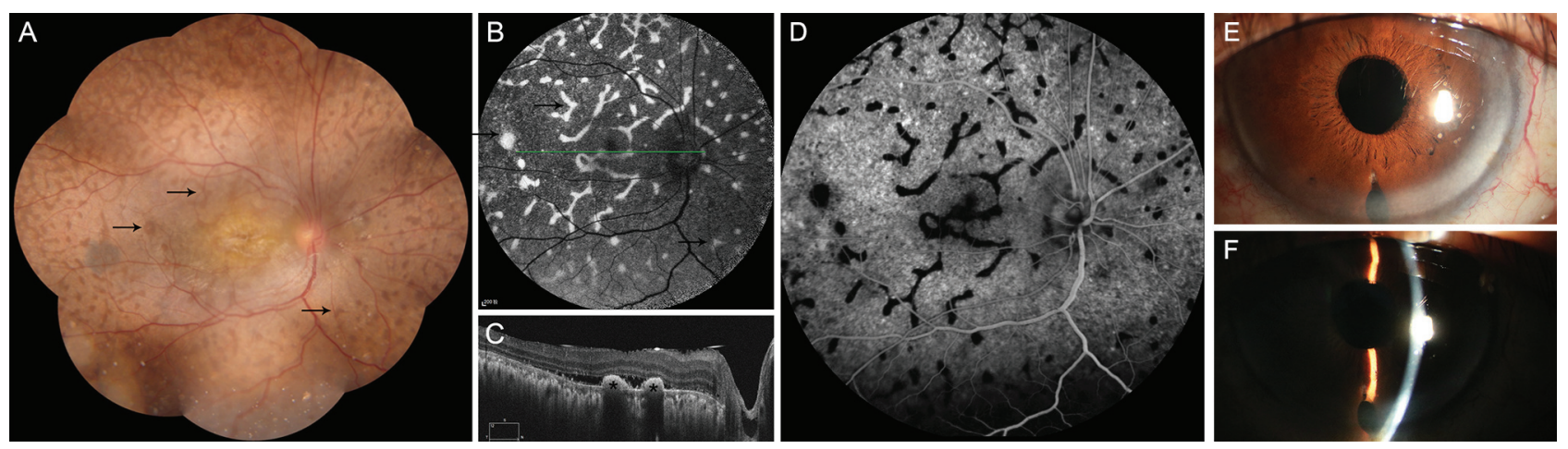

FIGURE 3 | The main clinical manifestation of the patient after surgery. (A) shows a mass of giraffe-like multifocal reddish patches (arrow) in the retina. These patches were consistent with increased autofluorescence (B, arrow), decreased fluorescence in early phase of FFA (D), and with multiple hyperreflective elevated lesions of the retinal pigment epithelium (C, asterisk), as revealed via ocular coherence tomography. It shows significantly deepening of the anterior chamber after surgery (E,F).
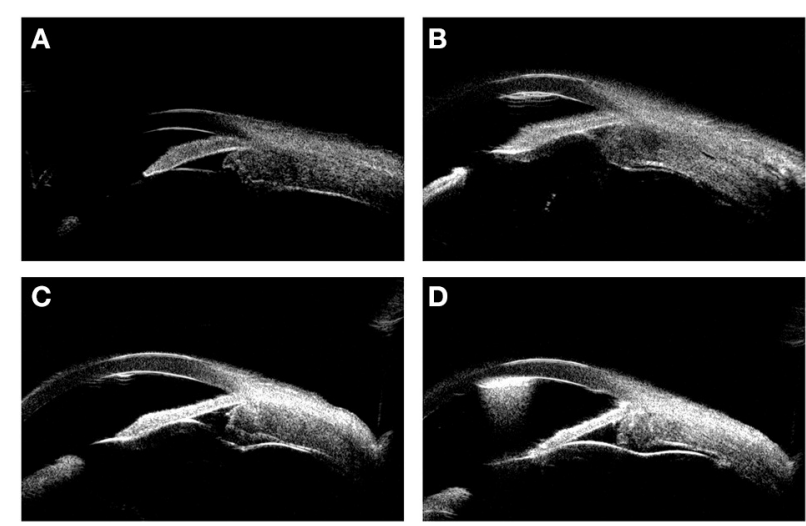

FIGURE 4 | The UBM images showing the thickness variation during the surgery. (A) shows the thickened ciliary body and choroid before the surgery. (B-D) show the thickness variation of ciliary body and choroid 1, 9, 18 months (6 months after rectectomy) after the surgery, respectively.

\section{DISCUSSION}

\section{Diagnosis}

Gass (6) first described the characteristics of BDUMP as multifocal red-orange subretinal patches with associated early hyperfluorescence in FA, scattered melanocytic tumors or thickening of the uveal tract, exudative retinal detachment, and rapid cataract progression. All of these features were present in our patient. As a type of paraneoplastic syndrome (22), BDUMP usually occurs in patients with systemic malignant disease, and the primary tumor is diagnosed after the presentation of BDUMP in nearly half of cases (3). In our case, the primary tumor was a rectal adenocarcinoma, and it was discovered more than 1 year after the appearance of BDUMP. No clear malignancy was found during treatment in the Department of Ophthalmology, and the patient was known only to have a history of subtotal pneumonectomy due to a pulmonary benign tumor. The diagnosis in this case was confusing to us, as the
BDUMP diagnosis was made without any medical history of a primary malignancy.

\section{Histopathological Examination}

According to the histopathological examination, biopsies from the pigmented intra-bulbar lesions showed predominantly spindle-shaped melanocytic cells with an occasional admixture of epithelial cells, but no or rare mitotic activity and no atypia (3). HE staining of biopsies collected from the choroid during surgery revealed a predominant spindle-shaped melanocytic cell mass, which was consistent with previous studies $(9,23)$. The immunohistochemical data for the biopsy provided useful information about the nature of the tissue. S-100 is normally present in cells derived from the neural crest (Schwann cells and melanocytes), chondrocytes, adipocytes, myoepithelial cells, macrophages, Langerhans cells, dendritic cells, and keratinocytes. S-100 can be found in melanomas, and, in some previous studies, was used as a biomarker for identifying melanocytes (24). In the present case, the positive expression of S-100 demonstrated that the tissue might have originated from melanocytes. Ki67 is a nuclear protein whose expression is strongly associated with tumor cell proliferation and growth $(25,26)$. Therefore, it is widely used in routine pathological investigations as a proliferation marker (26). In this case, the negative expression of Ki67 demonstrated the benign biological status of the tissue. When describing the characteristics of BDUMP, Gass (6) proposed that BDUMP be diagnosed differentially according to weather conditions resembled BDUMP before vs. after the development of multifocal pigmented choroidal tumors. BDUMP has been associated with B-cell lymphoma (20) and primary vitreoretinal lymphoma (21). Therefore, we conducted immunohistochemical staining against LCA, PAX-5, and SOX10. The PAX-5 gene is a member of the paired box (PAX) family of transcription factors. PAX proteins are important regulators in early development, and alterations in the expression of their genes are thought to contribute to neoplastic transformation. The PAX-5 gene encodes B-cell lineage specific activator protein (BSAP) (27), which is expressed at early, but not late, stages of B-cell differentiation. As its expression has also been detected 
in the developing central nervous system, PAX-5 gene products may not only play an important role in B-cell differentiation (28), but also in neural development. The deregulation of PAX5 transcription contributes to the pathogenesis of lymphomas and up to $97 \%$ of the Reed-Sternberg cells in patients with Hodgkin's lymphoma express Pax-5 (29). The SOX-10 gene encodes a member of the SOX (SRY-related HMG-box) family of transcription factors, which are involved in the regulation of embryonic development and determination of cell fate. Mutations in the SOX-10 gene have been associated with uveal melanoma (30). Because immunohistochemical staining against LCA, PAX-5, and SOX-10 was negative, we excluded the possibility of B-cell lymphoma and uveal melanoma.

\section{Cultured Melanocyte Elongation and Proliferation (CMEP) Factor}

Although the exact pathogenesis of BDUMP and the associated mechanisms of uveal and dermal melanocytic proliferation remain unclear, CMEP factor from the IgG-rich fraction of serum from patients with BDUMP was previously found to stimulate melanocytic proliferation (31). Specifically, human melanocytes grew when they were exposed to serum or plasma from patients with BDUMP. Treatment of other cells, such as human dermal fibroblasts, keratinocytes, and ovarian cancer cells, with plasma from BDUMP patients indicated that the proliferation was melanocyte selective. This observation was supported by Jansen (32), who reported two cases of BDUMP in whom serum was collected. The serum from the first patient was subjected to plasmapheresis and did not demonstrate proliferation of cultured human melanocytes. However, the serum from the second patient was evaluated prior to treatment with plasmapheresis and did induce proliferation. In theory, CMEP factor should not have been present in the serum from the first patient but should have been present in the serum from the second patient. When human melanocytes were exposed to serum from the BDUMP patients, only the serum from the second patient induced melanocytic proliferation, confirming the ability of an IgG factor in BDUMP patients to induce proliferation.

\section{Treatment}

Because systemic CMEP factor produced by systemic malignant diseases is considered to be involved in the pathogenesis of BDUMP, most previous case reports indicated that BDUMP treatment targeted the primary malignancies or metastases, including local resection, radiation, chemotherapy, or various combinations of these approaches $(3,14)$, and that visual symptoms and signs improved after the treatment. In our case, subretinal fluid was totally absorbed and the thickness of the

\section{REFERENCES}

1. Machemer R. [On the pathogenesis of the flat malignant melanoma]. Klin Monbl Augenheilkd. (1966) 148:641-52.

2. Lavine JA, Ramos MS, Wolk AM, Baynes K, Sharma S, Rachitskaya AV, et al. Heterogeneity of cultured melanocyte elongation and proliferation ciliary body and choroid decreased with improvement in the BCVA score 6 months after the rectectomy. Plasmapherisis or plasma exchange is another way to remove CMEP in the plasma, and these approaches have effectively improved the BCVA and exudative retinal detachment in some BDUMP patients $(12,23$, 32-36). In addition, anti-vascular endothelium growth factor agents and corticosteroids have been used to promote the absorption of subretinal fluid and improve the BCVA, although this treatment was not satisfactory $(15,35,37)$.

\section{Prognosis}

Although BDUMP is considered to be a benign proliferative disease, its prognosis is poor due to the primary malignancy, with an overall mean survival of 15.7 months after the presentation of BDUMP (3). Therefore, it is extremely important to establish diagnostic criteria enabling the timely detection of potential malignancies in patients with BDUMP.

\section{CONCLUSION}

The typical clinical manifestations of BDUMP are multifocal red-orange subretinal patches, scattered melanocytic tumors or thickening of the uveal tract, exudative retinal detachment, and rapid cataract progression. Detailed systemic examinations to identify potential primary malignancies should be performed in patients with suspected BDUMP to facilitate early treatment and improve prognoses. In the present case, rectal adenocarcinoma was associated with BDUMP for the first time.

\section{AUTHOR CONTRIBUTIONS}

NT and LW made substantial contributions to the drafting of the article and conducted revisions of the manuscript. NW made substantial contributions to the acquisition, analysis, and interpretation of the data for this work. $\mathrm{ZZ}$ made substantial contributions to the conception and design of the work. All authors contributed to the article and approved the submitted version.

\section{SUPPLEMENTARY MATERIAL}

The Supplementary Material for this article can be found online at: https://www.frontiersin.org/articles/10.3389/fmed. 2021.691686/full\#supplementary-material

Supplementary Figure 1 | Photos from the anterior segment examination, which showed obviously tortuous and dilated episcleral vessels (A-D for the right eye showing the temporal, superior, inferior, and nasal side, respectively; E-H for the left eye showing the temporal, superior, inferior, and nasal side, respectively). literature review. Acta Ophthalmol. (2017) 95:439-45. doi: 10.1111/aos. 13481 
4. Guo J, Tang W, Liu W, Zhou M, Chang Q, Jiang C, et al. A case report of ultrasonographic findings in bilateral diffuse uveal melanocytic proliferation. BMC Ophthalmol. (2020) 20:476. doi: 10.1186/s12886-020-01720-6

5. O’Day R, Michalova K, Campbell WG. Bilateral diffuse uveal melanocytic proliferation associated with bladder cancer: a novel imaging finding. Ophthalmic Surg Lasers Imaging Retina. (2019) 50:525-8. doi: 10.3928/23258160-20190806-10

6. Gass JD, Gieser RG, Wilkinson CP, Beahm DE, Pautler SE. Bilateral diffuse uveal melanocytic proliferation in patients with occult carcinoma. Arch Ophthalmol. (1990) 108:527-33. doi: 10.1001/archopht.1990.01070060075053

7. Jabbarpoor Bonyadi MH, Ownagh V, Rahimy E, Soheilian M. Giraffe or leopard spot chorioretinopathy as an outstanding finding: case report and literature review. Int Ophthalmol. (2019) 39:1405-12. doi: 10.1007/s10792-018-0948-5

8. Alasil T, Coady PA, Koudsi S, Mathur M, Materin MA. Bilateral diffuse uveal melanocytic proliferation: a case report. Retin Cases Brief Rep. (2017) 11:71-4. doi: 10.1097/ICB.0000000000000292

9. Duong HV, McLean IW, Beahm DE. Bilateral diffuse melanocytic proliferation associated with ovarian carcinoma and metastatic malignant amelanotic melanoma. Am J Ophthalmol. (2006) 142:693-5. doi: 10.1016/j.ajo.2006.04.059

10. Donovan JT, Prefontaine M, Gragoudas ES. Blindness as a consequence of a paraneoplastic syndrome in a woman with clear cell carcinoma of the ovary. Gynecol Oncol. (1999) 73:424-9. doi: 10.1006/gyno.1998.5332

11. van Noort BC, Keunen JEE, Schlingemann RO, Marinkovic M. Long survival and preservation of good visual acuity in a patient with bilateral diffuse uveal melanocytic proliferation. Ocul Oncol Pathol. (2019) 5:758. doi: 10.1159/000488454

12. Schelvergem KV, Wirix M, Nijs I, Leys A. Bilateral diffuse uveal melanocytic proliferation with good clinical response to plasmapheresis and treatment of the primary tumor. Retin Cases Brief Rep. (2015) 9:1068. doi: 10.1097/ICB.0000000000000104

13. Yu S, Ikeda T, Ikeda N, Mimura O, Sato K. Coloration of fundus lesions in bilateral diffuse uveal melanocytic proliferation. Jpn J Ophthalmol. (2003) 47:612-5. doi: 10.1016/j.jjo.2003.09.003

14. Luo M, Chen Z, Luo Y, Zhao L, Dai R, Zhong Y. Diagnosis of bilateral diffuse uveal melanocytic proliferation unveils primary gastric adenocarcinoma: a case report. BMC Ophthalmol. (2020) 20:113. doi: 10.1186/s12886-020-01376-2

15. Dolz-Marco R, Vilaplana F, Gallego-Pinazo R, Freund KB. Delayedonset bilateral diffuse uveal melanocytic proliferation associated with gastric adenocarcinoma. Retin Cases Brief Rep. (2017) 11(Suppl. 1):S1826. doi: 10.1097/ICB.0000000000000429

16. Roblain AS, Malaise D, Lepiece G, Locht B, Rakic JM. [Bilateral diffuse uveal melanocytic proliferation: a rare paraneoplasic syndrome to be diagnosed early]. Rev Med Liege. (2021) 76:160-5.

17. Lentzsch AM, Siggel R, Spital C, Holtick U, Liakopoulos S. Long-time followup of asymmetric bilateral diffuse uveal melanocytic proliferation (BDUMP) in a patient with metastasized urothelial carcinoma. Retin Cases Brief Rep. (2021). doi: 10.1097/ICB.0000000000001129. [Epub ahead of print].

18. Breazzano MP, Bacci T, Wang H, Francis JH, Yannuzzi LA. Bacillary layer detachment in bilateral diffuse uveal melanocytic proliferation masquerading as neovascular AMD. Ophthalmic Surg Lasers Imaging Retina. (2020) 51:4137. doi: 10.3928/23258160-20200702-07

19. O’Neal KD, Butnor KJ, Perkinson KR, Proia AD. Bilateral diffuse uveal melanocytic proliferation associated with pancreatic carcinoma: a case report and literature review of this paraneoplastic syndrome. Surv Ophthalmol. (2003) 48:613-25. doi: 10.1016/j.survophthal.2003.08.005

20. Pefkianaki M, Agrawal R, Desai P, Pavesio C, Sagoo MS. Bilateral Diffuse Uveal Melanocytic Proliferation (BDUMP) associated with B-cell lymphoma: report of a rare case. BMC Cancer. (2015) 15:23. doi: 10.1186/s12885-015-1020-8

21. Leskov I, Lyon AT, Jampol LM. Diffuse uveal melanocytic proliferation with primary vitreoretinal lymphoma. JAMA Ophthalmol. (2019) 137:8347. doi: 10.1001/jamaophthalmol.2019.0905

22. Bussat A, Langner-Lemercier S, Salmon A, Mouriaux F. Paraneoplastic syndromes in ophthalmology. J Fr Ophtalmol. (2018) 41:e1815. doi: 10.1016/j.jfo.2018.03.002
23. Pulido JS, Flotte TJ, Raja H, Miles S, Winters JL, Niles R, et al. Dermal and conjunctival melanocytic proliferations in diffuse uveal melanocytic proliferation. Eye. (2013) 27:1058-62. doi: 10.1038/eye.2013.131

24. Chen C, Jing W, Gulati P, Vargas H, French SW. Melanocytic differentiation in a solid pseudopapillary tumor of the pancreas. J Gastroenterol. (2004) 39:579-83. doi: 10.1007/s00535-004-1346-5

25. Acay RR, Felizzola CR, de Araujo N, de Sousa SO. Evaluation of proliferative potential in oral lichen planus and oral lichenoid lesions using immunohistochemical expression of p53 and Ki67. Oral Oncol. (2006) 42:47580. doi: 10.1016/j.oraloncology.2005.09.012

26. Denkert C, Budczies J, von Minckwitz G, Wienert S, Loibl S, Klauschen F. Strategies for developing Ki67 as a useful biomarker in breast cancer. Breast. (2015) 24(Suppl. 2):S67-72. doi: 10.1016/j.breast.2015.0 7.017

27. Michaelson JS, Singh M, Birshtein BK. B cell lineage-specific activator protein (BSAP). A player at multiple stages of B cell development. J Immunol. (1996) 156:2349-51.

28. O’Brien P, Morin P Jr, Ouellette RJ, Robichaud GA. The Pax-5 gene: a pluripotent regulator of B-cell differentiation and cancer disease. Cancer Res. (2011) 71:7345-50. doi: 10.1158/0008-5472.CAN-11-1874

29. Torlakovic E, Torlakovic G, Nguyen PL, Brunning RD, Delabie J. The value of anti-pax-5 immunostaining in routinely fixed and paraffin-embedded sections: a novel pan pre-B and B-cell marker. Am J Surg Pathol. (2002) 26:1343-50. doi: 10.1097/00000478-200210000-00011

30. Das D, Kaur I, Ali MJ, Biswas NK, Das S, Kumar S, et al. Exome sequencing reveals the likely involvement of SOX10 in uveal melanoma. Optom Vis Sci. (2014) 91:e185-92. doi: 10.1097/OPX.0000000000000309

31. Miles SL, Niles RM, Pittock S, Vile R, Davies J, Winters JL, et al. A factor found in the IgG fraction of serum of patients with paraneoplastic bilateral diffuse uveal melanocytic proliferation causes proliferation of cultured human melanocytes. Retina. (2012) 32:1959-66. doi: 10.1097/IAE.0b013e3182618bab

32. Jansen JC, Van Calster J, Pulido JS, Miles SL, Vile RG, Van Bergen $\mathrm{T}$, et al. Early diagnosis and successful treatment of paraneoplastic melanocytic proliferation. $\mathrm{Br} \quad J$ Ophthalmol. (2015) 99:943-8. doi: 10.1136/bjophthalmol-2014-305893

33. Mets RB, Golchet P, Adamus G, Anitori R, Wilson D, Shaw J, et al. Bilateral diffuse uveal melanocytic proliferation with a positive ophthalmoscopic and visual response to plasmapheresis. Arch Ophthalmol. (2011) 129:12358. doi: 10.1001/archophthalmol.2011.277

34. Navajas EV, Simpson ER, Krema H, Hammoudi DS, Weisbrod D, Bernardini $\mathrm{M}$, et al. Cancer-associated nummular loss of RPE: expanding the clinical spectrum of bilateral diffuse uveal melanocytic proliferation. Ophthalmic Surg Lasers Imaging. (2011) 42:e103-6. doi: 10.3928/1542887720111020-02

35. Jaben EA, Pulido JS, Pittock S, Markovic S, Winters JL. The potential role of plasma exchange as a treatment for bilateral diffuse uveal melanocytic proliferation: a report of two cases. J Clin Apher. (2011) 26:35661. doi: 10.1002/jca.20310

36. Alrashidi S, Aziz AA, Krema H. Bilateral diffuse uveal melanocytic proliferation: a management dilemma. BMJ Case Rep. (2014) 2014. doi: 10.1136/bcr-2014-204387

37. Saito W, Kase S, Yoshida K, Ohguro H, Yokoi M, Iwaki H, et al. Bilateral diffuse uveal melanocytic proliferation in a patient with cancer-associated retinopathy. Am J Ophthalmol. (2005) 140:942-5. doi: 10.1016/j.ajo.2005.05.048

Conflict of Interest: The authors declare that the research was conducted in the absence of any commercial or financial relationships that could be construed as a potential conflict of interest.

Copyright $\odot 2021$ Tong, Wang, Wang and Zhou. This is an open-access article distributed under the terms of the Creative Commons Attribution License (CC BY). The use, distribution or reproduction in other forums is permitted, provided the original author(s) and the copyright owner(s) are credited and that the original publication in this journal is cited, in accordance with accepted academic practice. No use, distribution or reproduction is permitted which does not comply with these terms. 